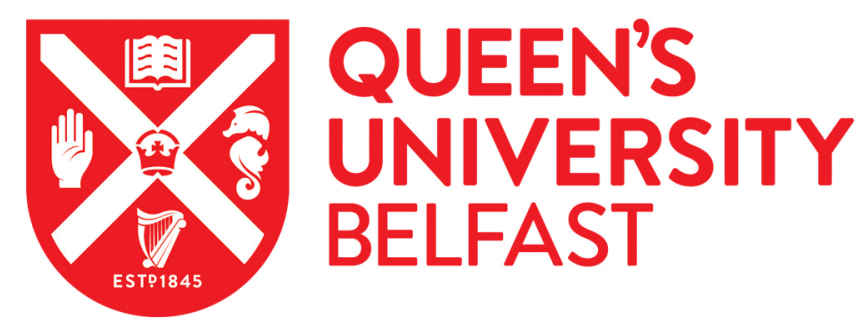

\title{
Nanoscale Hybrid Coating Enables Multifunctional Tissue Scaffold for Potential Multimodal Therapeutic Applications
}

Guo, Z., Jiang, N., Moore, J., McCoy, C., Ziminska, M., Rafferty, C., Sarri, G., Hamilton, A., Li, Y., Zhang, L., Zhu, S., \& Sun, D. (2019). Nanoscale Hybrid Coating Enables Multifunctional Tissue Scaffold for Potential Multimodal Therapeutic Applications. ACS Applied Materials and Interfaces, 11(30), 27269.

https://doi.org/10.1021/acsami.9b04278

Published in:

ACS Applied Materials and Interfaces

Document Version:

Peer reviewed version

Queen's University Belfast - Research Portal:

Link to publication record in Queen's University Belfast Research Portal

Publisher rights

Copyright 2019 ACS. This work is made available online in accordance with the publisher's policies. Please refer to any applicable terms of use of the publisher.

\section{General rights}

Copyright for the publications made accessible via the Queen's University Belfast Research Portal is retained by the author(s) and / or other copyright owners and it is a condition of accessing these publications that users recognise and abide by the legal requirements associated with these rights.

Take down policy

The Research Portal is Queen's institutional repository that provides access to Queen's research output. Every effort has been made to ensure that content in the Research Portal does not infringe any person's rights, or applicable UK laws. If you discover content in the Research Portal that you believe breaches copyright or violates any law, please contact openaccess@qub.ac.uk. 


\title{
Nanoscale hybrid coating enables multi-functional tissue scaffold for potential multi-modal therapeutic applications
}

Zhijun Guo ${ }^{1,3 \dagger}$, Nan Jiang ${ }^{2 \dagger}$, Jessica Moore ${ }^{4}$, Colin P. McCoy ${ }^{4}$, Monika Ziminska ${ }^{4}$, Cormac Rafferty ${ }^{5}$, Gianluca Sarri ${ }^{5}$, Andrew R. Hamilton ${ }^{6}$, Yubao Li $^{3}$, Li Zhang ${ }^{3}$, Songsong $\mathrm{Zhu}^{2 *}$, Dan $\operatorname{Sun}^{1 *}$

1. School of Mechanical \& Aerospace Engineering, Queens University Belfast, Belfast, BT9 5AH, UK

2. State Key Laboratory of Oral Diseases, \& National Clinical Research Center for Oral Disease, \& West China Hospital of Stomatology, Sichuan University, Chengdu, China

3. Analytical and Testing Center, Sichuan University, Chengdu 610064, China

4. School of Pharmacy, Queens University Belfast, Belfast, BT9 7BL, UK

5. School of Mathematics and Physics, Queens University Belfast, Belfast, BT7 1NN

6. Faculty Engineering and the Environment University of Southampton, SO17 1BJ, UK

*Corresponding authors: $\underline{\text { d.sun@ @ub.ac.uk; zss_1977@163.com }}$

${ }^{\dagger}$ First and second author contributed equally

\begin{abstract}
Through a nature-inspired layer-by-layer assembly process, we developed a unique multifunctional tissue scaffold that consists of porous polyurethane substrate and nanoscale chitosan/ graphene oxide hybrid coating. Alternative layers of drug laden chitosan and graphene oxide nanosheets were held together through strong electrostatic interaction, giving rise to a robust multilayer architecture with control over structural element orientation and chemical composition at nanoscale. Combined $\mathrm{pH}$-controlled co-delivery of multiple therapeutic agents and photothermal therapy have achieved by our scaffold system. The new
\end{abstract}


platform technology can be generalized to produce other tissue scaffold systems and may enable potential multi-modal therapeutic applications such as bone cancer managements.

Keywords: hybrid coating, tissue scaffold, multi-modal therapy, co-drug delivery, photothermal therapy

\section{Introduction}

Multi-modal therapeutics are highly desirable under certain challenging clinical settings such as management of bone cancers (such as osteosarcoma and bone metastases). Traditional treatment of bone cancers involves surgical removal of cancerous tissue, the use of radiotherapy / chemotherapy (cancer drugs) to manage pain and control tumor growth / recurrence. ${ }^{1}$ Other treatments may also be required for combatting surgery-induced infections and promoting functional recovery. Bone scaffold that can fill the large bone defects while offering therapeutic functions such as controlled drug release for symptom (anti-tumoral, pain) relief, infection control, preventing cancer recurrence and promoting bone regeneration, are promising treatment protocol for reducing the risk of systemic effects and improve patient's quality of life.

To date, research on multi-functional bone scaffolds that offer multi-modal therapeutics is still in its infancy. Wong et al. ${ }^{2}$ and Palamà et al., ${ }^{3}$ developed drug-laden polycaprolactone (PCL) scaffolds for the postsurgical care of osteosarcoma. The scaffold porosity was achieved by press-molding or solvent casting / particulate leaching technique; however it is difficult to control the pore size/interconnection and porosity of the scaffolds. Zhang et al., ${ }^{4}$ printed three dimensional (3D) PCL composite scaffold containing mesoporous bioactive glass and $\mathrm{Fe}_{3} \mathrm{O}_{4}$ nanoparticles (NPs). The magnetic scaffolds have uniform and continuous pore structure with improved mechanical properties (compressive strength and toughness) and can offer sustained cancer drug release. However, harsh/hazardous solvents are necessary for the preparation of the printable materials, which may present challenges on the drug bioactivity and in vivo toxicity of the scaffold. In addition, despite the biocompatibility of magnetic $\mathrm{Fe}_{3} \mathrm{O}_{4} \mathrm{NPs}$, they could be toxic to cells when concentrations are more than $0.05 \mathrm{mg} / \mathrm{L} .{ }^{4} \mathrm{In}$ recent years, photothermal therapy (PTT) which employs heat generated from absorbed near-infrared (NIR) 
light to ablate cancer, has developed rapidly in tumor treatment. Various photothermal agents, including gold nanoparticles ${ }^{5}$ and other emerging nanostructures such as $\mathrm{MoS}_{2}$ nanosheets ${ }^{6}$ and $\mathrm{CuFeSe}_{2}{ }^{7}$ nanocrystals have been incorporated into bone scaffolds to enable photothermal therapy in bone cancer treatment. A recent study also reported a 3D printed bifunctional graphene oxide (GO)-modified $\beta$-tricalcium phosphate composite scaffold with combined photothermal effect and significantly improved bone-forming ability. ${ }^{8}$ However, to the best of our knowledge, there has been no 3D bone scaffold available to date to provide combined PPT and drug delivery, especially co-delivery of multi-therapeutic agents to meet the demanding requirements in bone cancer management.

In this study, we designed and fabricated a unique scaffold system which consists of porous polyurethane (PU) foam substrate with drug laden graphene oxide (GO) nanosheet /chitosan (CS) hybrid coatings. PU can be produced with a wide range of mechanical and physical properties, depending on its composition and synthesis procedure. ${ }^{9,10}$ Researchers in the past have successfully modified the physiomechanical properties of PU to fit specific tissue engineering applications. ${ }^{11,12}$ In this study, biocompatible and biodegradable polyurethane (PU) with open porosity $>70 \%$ was used as the model template in this study as it mimics the trabecular bone structures ${ }^{13-15}$. GO nanosheets have high specific surface area with abundant negatively charged surface functions and has been used widely for the loading / delivery of various therapeutic agents ${ }^{16,17}$ and sensing of biological species. ${ }^{18}$ Being an electroactive material, GO can also achieve electrically-induced drug-release ${ }^{8}$ and cell growth. ${ }^{19}$ On the other hand, GO is one of the most promising candidates for photothermal cancer therapy due to its strong NIR absorbance, high-photothermal-conversion efficiency, excellent thermal conductivity, and strong potential in stimulating bone regeneration. ${ }^{19}$ Biocompatible and degradable CS was chosen as the polycation polymer and its abundant surface functional groups (hydroxyl and amino) and positive surface charge can enable the loading of various therapeutic agents (e.g, anti-cancer drugs, ${ }^{20}$ growth factors, ${ }^{21}$ anti-microbial agents, ${ }^{22}$ etc.) and interact electrostatically with GO to form a robust coating. We hypothesize that the proposed hybrid coating system will have the combined advantage from its constituent materials and will provide several important functionalities to the tissue scaffold, including delivery of multiple 
therapeutic payloads, providing on-demand local photo-thermal therapy and offering excellent biocompatibility.

\section{Results and discussion}

\subsection{Materials characterization}

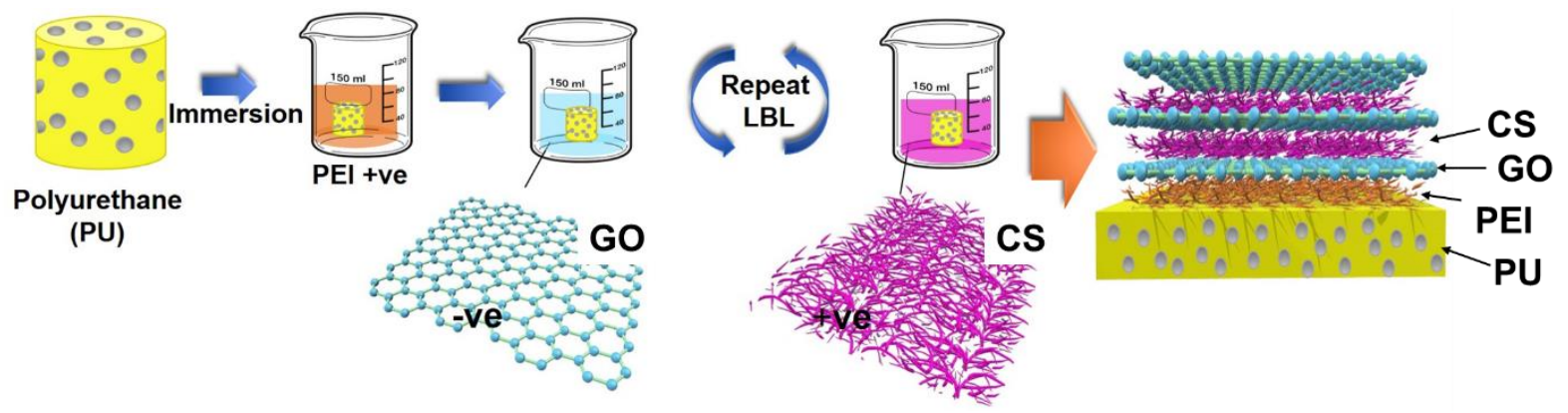

Scheme 1. Layer-by-layer assembly of CS/GO hybrid coating on porous PU scaffold

Here we deployed a nature-inspired layer-by-layer assembly (LBL) process to produce hierarchical multilayered materials that mimic the structure of nacre and bones. ${ }^{23}$ Alternative layers of oppositely charged organic/inorganic building blocks were deposited onto an organic substrate forming a multi-layer hybrid coating, see Scheme 1 (more detailed description see Experimental Methods). Alternative layers of CS and GO were held together through a strong electrostatic interaction, giving rise to a multilayer architecture with control over structural element orientation and chemical composition at nanoscale. As both GO and CS can be loaded with model drugs such as fluorescein sodium (FL), methyl blue (MB) or Ag nanoparticles (AgNPs), different coating systems such as (GO-CS $)_{n}$, (n denotes number of bilayers), single or dual drug loaded $(\mathrm{GO} / \mathrm{FL}-\mathrm{CS})_{\mathrm{n}},(\mathrm{GO}-\mathrm{CS} / \mathrm{MB})_{\mathrm{n}},(\mathrm{GO} / \mathrm{FL}-\mathrm{CS} / \mathrm{MB})_{\mathrm{n}}$ and $(\mathrm{GO}-\mathrm{CS} / \mathrm{AgNPs})_{\mathrm{n}}$, have been produced following the similar LBL process. Theoretically, the coating thickness and choice of drugs for the hybrid coating can be customized according to the patient's specific disease conditions. The optical image in Figure 1 (a) shows that the scaffold color turns darker with increasing number of CS/GO bilayers, indicating a greater amount of GO has been successfully deposited. The SEM insets suggest that the coating process has no effect on the 
high level of interconnectivity of the porous scaffold. Figure 1 (b) shows the cross-sectional SEM image of a typical coated foam strut and Figure 1 (c) is the close-up image showing the morphology of a 30 bi-layer hybrid coating. The thickness of a 5-bilayer coating is $\sim 0.65 \pm$ $0.32 \mu \mathrm{m}$ and $\sim 3.90 \pm 1.37 \mu \mathrm{m}$ for a 30-bilayer coating, according to SEM observation of different samples. The coating thickness was also confirmed by AFM (Figure 1 (d)). The result is consistent with the SEM analysis, where the average thickness for each bi-layer is $\sim 130 \pm$ $20 \mathrm{~nm}$.
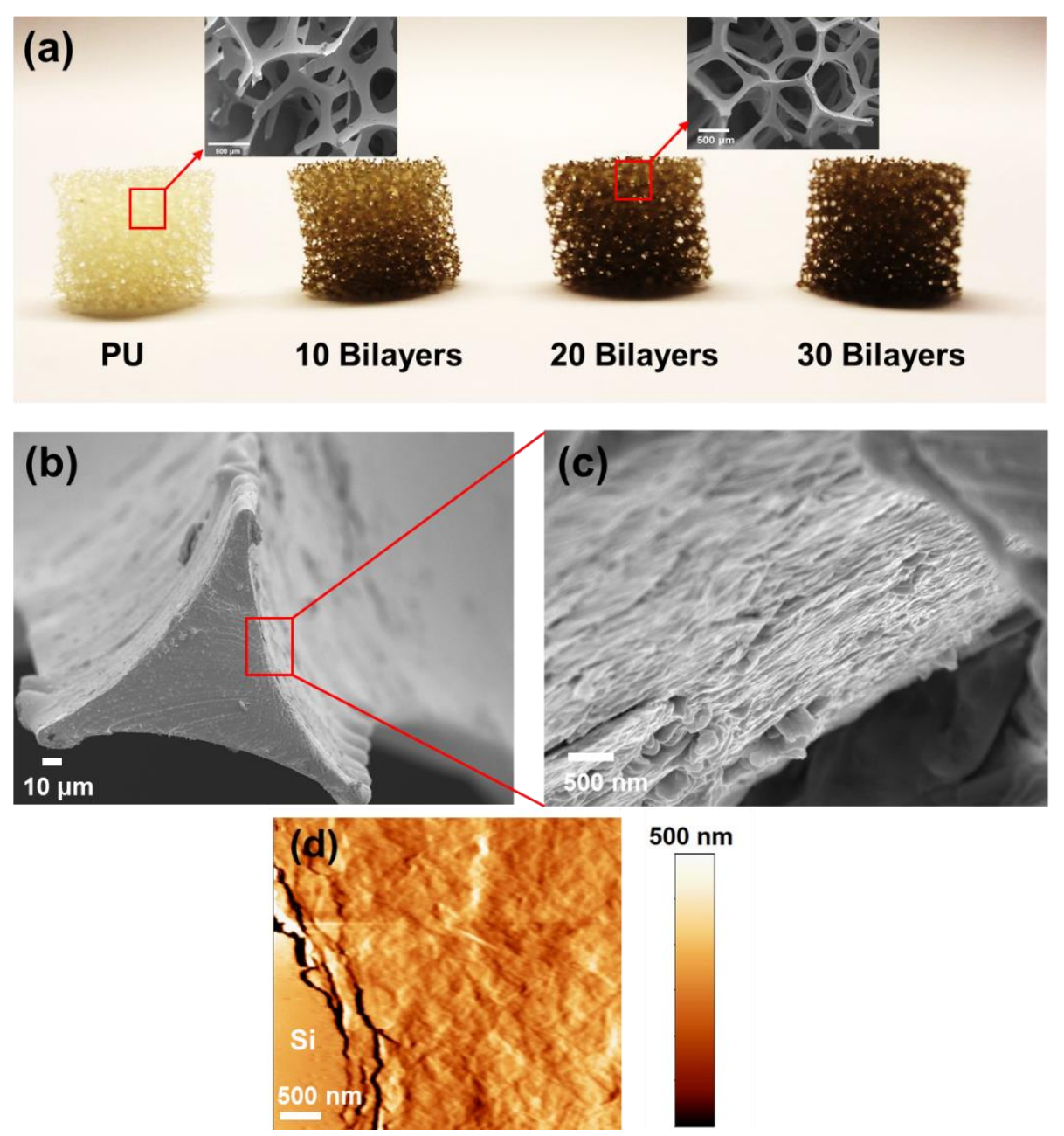

Figure 1. (a) Optical images of PU scaffolds with different number of bilayer coatings; inset: SEM image showing the scaffold microstructures before (top left) and after (top right) the coating deposition (b) cross-sectional SEM image of a coated foam strut (c) high magnification SEM image showing coating with 30 bilayers, (d) AFM image of 3 bilayers deposited on a Si substrate. 

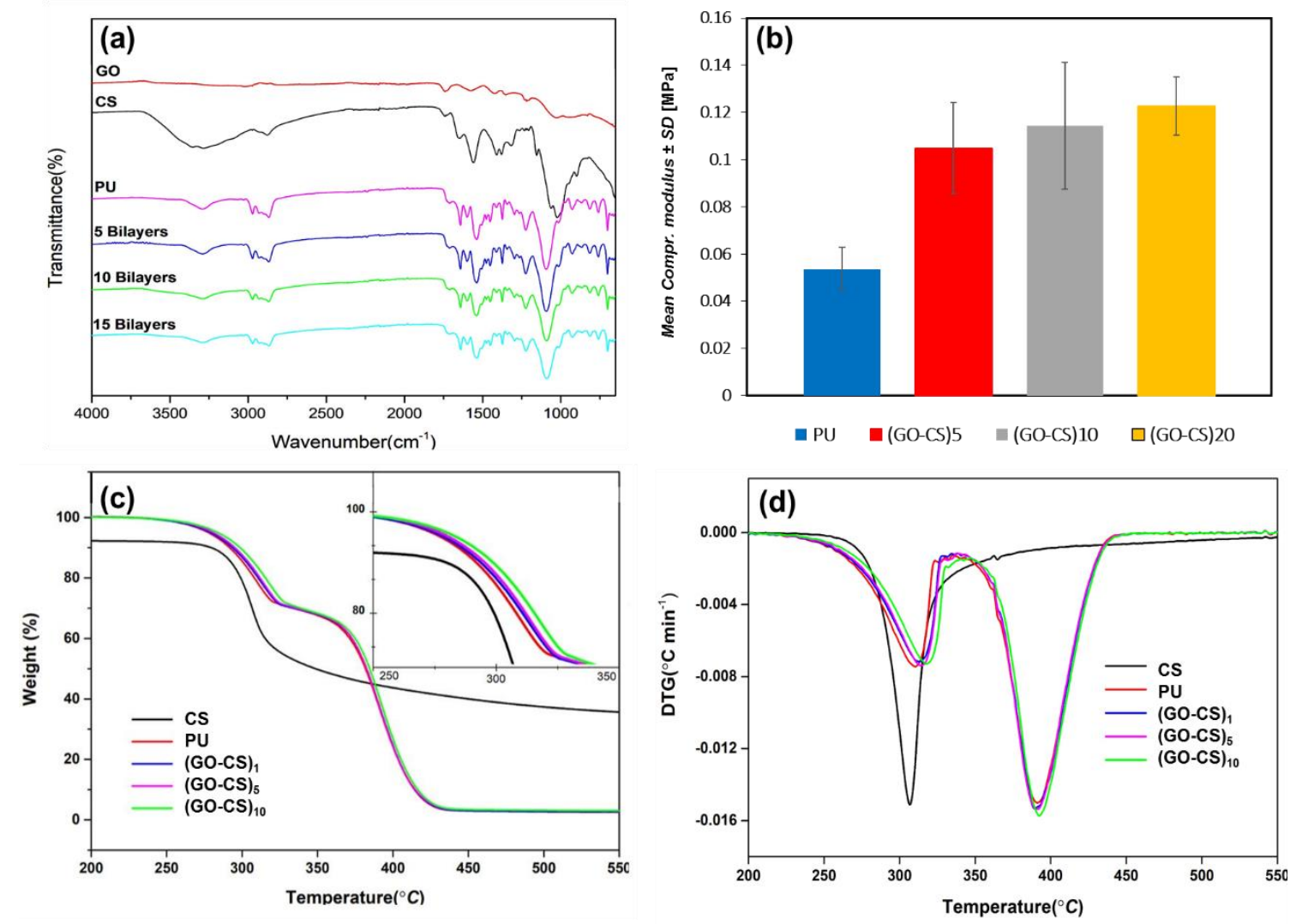

Figure 2. (a) FTIR spectra, (b) Mean compressive elastic modulus of scaffold (average taken from 5 scaffolds for each type of scaffold) with different number of bilayers (c) TGA, inset: magnification of the $5 \%$ weight loss, heat rate $10{ }^{\circ} \mathrm{C} \mathrm{min}^{-1}$ and (d) DTG spectra of PU scaffold with and without hybrid coatings.

Figure 2 (a) shows the FTIR spectra of the scaffold with different coating thicknesses. The gradually decreased PU characteristic peak intensity indicates the increased coating thickness as the LBL assembly process continues (Figure 2a). Compressive mechanical testing within the elastic range of the scaffold samples was conducted and an increasing trend in modulus was observed as the number of coated bilayers increased from 5 to 20, see Figure 2 (b). The compressive modulus for samples with 20 bilayer coating $(0.123 \pm 0.0122 \mathrm{MPa})$ was double that of the control PU samples $(0.053 \pm 0.0091 \mathrm{MPa})$ without affecting the interconnectivity of PU scaffold (See Figure 1 (a) SEM insets). While the compressive modulus of the PU foam and that of the subsequent coated scaffolds are not comparable to natural bones, the main focus of this study is to establish a platform technology for fabrication 
of multifunctional coatings. The technique can actually be generalized and applied to a wider range of surfaces (such as $\mathrm{Si}$ ) and different scaffold substrates (such as mechanically stronger 3D printed PU scaffold, see Figure S1 in Supporting Information). The thermal and thermooxidative stability of the scaffolds were studied by thermogravimetric analysis (TGA) in a nitrogen atmosphere. Figure 2 (c) and Figure 2 (d) shows the TGA and DTG spectra of scaffolds with and without coatings. Pure CS exhibits about $7.5 \%$ weight loss in the temperature range of $50-140{ }^{\circ} \mathrm{C}$ due to the evaporation of the absorbed water. The major weight loss of $57.9 \%$ between $250-400{ }^{\circ} \mathrm{C}$ is due to the degradation of polysaccharide units, which is in accordance with previous reports. ${ }^{18,24-28}$ The TGA curve of pristine PU foam demonstrated two main thermal events ${ }^{29}$ : 1) $28.4 \%$ weight loss around $210-330^{\circ} \mathrm{C}$, which is related to liberation of di-isocyanates due to depolymerization of the urethane and the disubstituted urea groups, 2) $97.63 \%$ weight loss around $340-600{ }^{\circ} \mathrm{C}$, which can be associated to the decomposition of the remaining polyether chain. These two thermal events are consistent with the two peaks in Figure 3(d). From Figure 3(c) inset, it can be seen that the temperature at 5\% weight loss $\left(\mathrm{T}_{5} \%\right)$ increased slightly with increasing number of bilayers when compared to that of the pure PU, and peak $\mathrm{T}_{5} \%$ in DTG and peak at $\mathrm{T}_{380^{\circ} \mathrm{C}}$ (Figure 2 (d)) red-shifted indicating improved coating thermal stability with increasing coating thickness. The coated samples presented almost similar weight loss but higher coating thermal stability for the temperature range $200-500{ }^{\circ} \mathrm{C}$. Comparing to pristine PU samples (residue 2.3622\%), more solid residue (2.847\%) remained for coated-PU which can be ascribed to the CS residual. The improved coating thermal stability may be a result of the excellent thermal stability of $\mathrm{GO}^{25}$ and the strong interaction between CS and GO which restricts the polymer motion on PU surface during the heating. ${ }^{30}$

To investigate the electrical properties of the hybrid coating, Si wafer was deployed as a model substrate to facilitate measurements. The electrical resistance of the hybrid coating was measured under ambient conditions using an Agilent 34450A51/2 Digital Mustimeter following a standard four terminal set-up. ${ }^{31}$ The electrical conductivity of (GO-CS) ${ }_{10}$ was $0.056 \mathrm{~S} / \mathrm{m}$, which is comparable to other CS/carbon system ${ }^{[41-42]}$ reported in the literature and is within the conductivity range of natural tissues such as ventricular muscle, blood, skeletal muscle 
(0.03-0.6 S/m), ${ }^{25,32,33}$ and brain, ${ }^{34}$ see Table 1. Such feature may enable the application of our coated scaffold for electrically induced sensing, ${ }^{35}$ drug release,${ }^{24}$ and tissue regeneration, ${ }^{18,36,37}$ etc.

Table 1. Comparison of electrical conductivity with materials reported in the literature

\begin{tabular}{|c|c|}
\hline Samples & Conductivity (S/m) \\
\hline CS/carbon (dry) ${ }^{25}$ & $0.25 \pm 0.09$ \\
\hline CS/carbon (hydrated) 25 & $0.04 \pm 0.02$ \\
\hline Ventricular muscle ${ }^{33}$ & 0.03 \\
\hline Body 25 & 0.2 \\
\hline Blood $^{25}$ & 0.6 \\
\hline Brain $^{34}$ & 0.12 \\
\hline Skeletal muscle 32 & 0.125 \\
\hline $\mathrm{CS}(\mathrm{dry})^{38}$ & $7.4 \times 10^{-09} \pm 1.0 \times 10^{-09}$ \\
\hline CS (hydrated) ${ }^{39}$ & $0.03 \pm 0.02$ \\
\hline This study: (GO-CS) 10 & 0.056 \\
\hline
\end{tabular}

\subsection{Functional characterization}

\subsubsection{Single drug release from GO}

Model drug fluorescein sodium (FL) can be loaded onto GO through formation of hydrogen bonding. ${ }^{40}$ The overall zeta potential of GO/FL is $-39.5 \mathrm{mV}$ as is shown in Figure 3(a). The molecular weight of FL is $376 \mathrm{~g} \mathrm{~mol}^{-1}$, similar to several important drugs such as the cancer drug cisplatin, the non-steroidal anti-inflammatory indomethacin, as well as various bone regeneration growth factors (FTY20, Sphingosine-1 phosphate , Vitamin D3, etc). ${ }^{41}$ When dissolved in neutral aqueous medium, FL gives a distinct absorption peak between 450 to $500 \mathrm{~nm}$ which can be detected by UV-vis spectroscopy (see Figure 3(b)). Figure 3(c) shows an initial burst release followed by stable release of FL $(\sim 65 \%)$ in the neutral aqueous environment over the first $\sim 12 \mathrm{~h}$ of immersion. From $12 \mathrm{~h}$ to $240 \mathrm{~h}$ the cumulative release 
profile remains stable $(\sim 65 \%)$. In contrast, there was no FL release detected under $\mathrm{pH} 4$ for up to 240 h. GO is hydrophilic and soluble under $\mathrm{pH}$ 7.4. Under $\mathrm{pH} 4$, it becomes less hydrophilic and tends to aggregate, and hence may impede the drug release. ${ }^{38,42}$ On the other hand, GO has much stronger negatively charged surface under neutral condition due to the ionization of the hydroxyl and carboxyl groups, ${ }^{43}$ see Figure S2. The greater negative surface charge density on the GO under $\mathrm{pH} 7.4$ may repel the FL as there are two negative charges spread over four $\mathrm{O}$ atoms in its structure. ${ }^{44}$
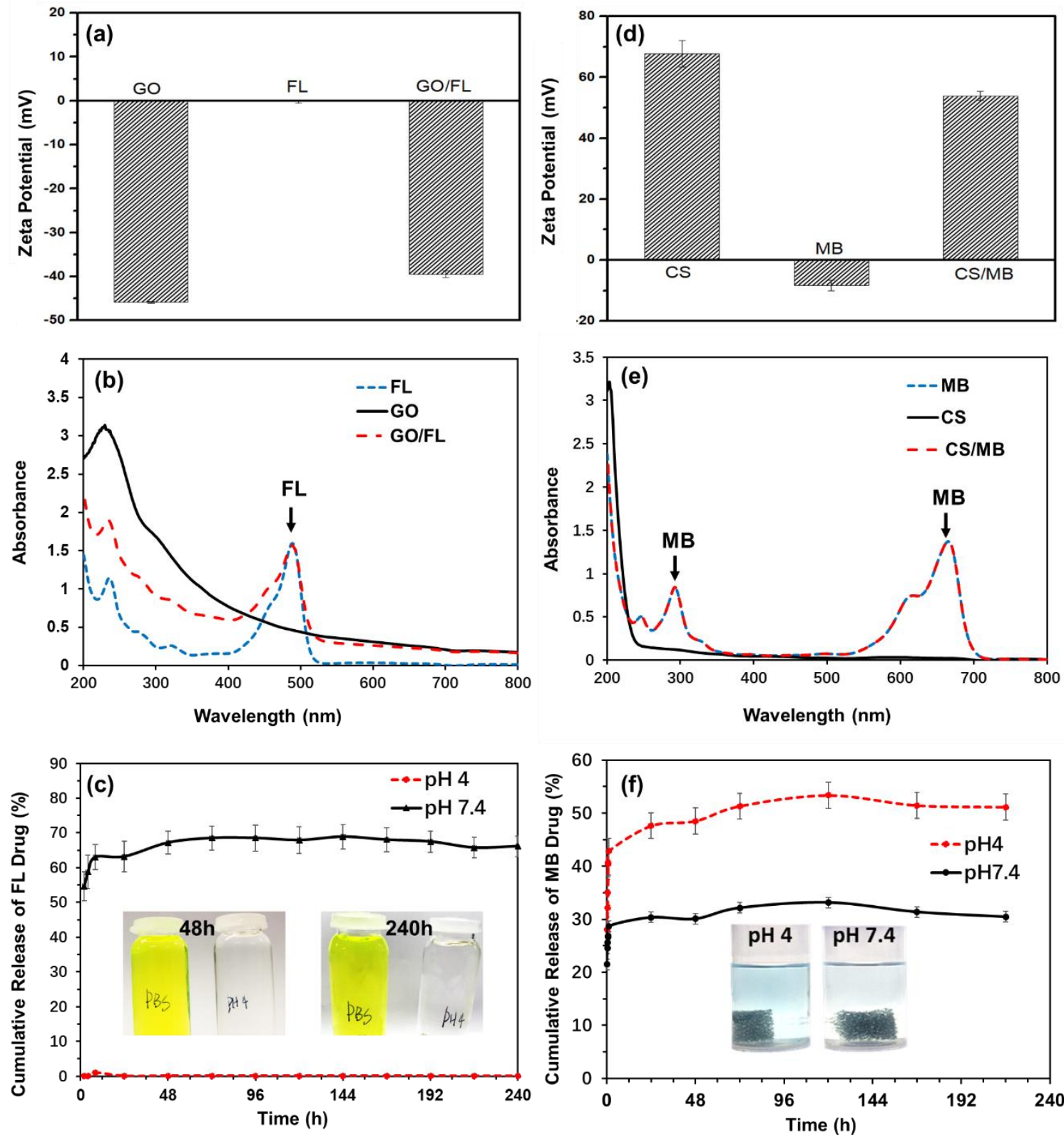
Figure 3. (a) Zeta potential of as-prepared GO, FL and GO/FL, (b) UV-vis spectra of asprepared GO, FL and GO/FL, (c) cumulative release (\%) of FL from (GO/FL-CS) $)_{20} \mathrm{PU}$ scaffold under neutral ( $\mathrm{pH} 7.4)$ and acidic $(\mathrm{pH} 4)$ conditions, (d) zeta potential of as-prepared $\mathrm{CS}, \mathrm{MB}$ and CS/MB, (e) UV-vis spectra of as-prepared CS, MB and CS/MB, (f) cumulative release $(\%)$ of $\mathrm{MB}$ from $(\mathrm{GO}-\mathrm{CS} / \mathrm{MB})_{5}$ scaffold under neutral $(\mathrm{pH} 7.4)$ and acidic $(\mathrm{pH} 4)$ environment.

\subsubsection{Single drug release from $\mathrm{CS}$}

Methylene blue (MB) was selected as a model drug to investigate the release profile from the scaffold containing drug-loaded CS. MB is negatively charged and has a molecular weight similar to that of many low-molecular weight drugs and growth factors used for bone regeneration. ${ }^{45}$ Figure 3 (d) shows the zeta potential of as-prepared MB, CS, and MB loaded CS (CS/MB). Through electrostatic interaction, MB was successfully loaded onto CS, giving $\mathrm{CS} / \mathrm{MB}$ an overall zeta potential of $53.9 \mathrm{mV}$. Figure 3 (e) shows the UV-vis spectra of asprepared CS, MB and CS/MB, respectively. The spectra of CS/MB overlaps with that of the MB, showing strong characteristic peaks at $668 \mathrm{~nm}$ and $292 \mathrm{~nm}$, which indicates the successful loading of MB. The effect of $\mathrm{pH}$ on the $\mathrm{MB}$ release profile has also been investigated for (GO$\mathrm{CS} / \mathrm{MB})_{5}$ samples immersed in acidic $(\mathrm{pH} 4)$ and neutral ( $\left.\mathrm{pH} 7.4\right)$ aqueous solutions, respectively. From Figure 3 (f), it can be seen that the release of MB under both $\mathrm{pH}$ conditions undergoes a burst release at the initial stage, after which the drug concentration stabilized at averages of around 55\% under $\mathrm{pH} 4$ and $30 \%$ under $\mathrm{pH}$ 7. It has previously been reported that the swelling of CS plays an important role in the drug release process, and the release is mainly controlled by diffusion ${ }^{46}$. The $\mathrm{pH}$ dependent $\mathrm{MB}$ release can be attributed to the surface protonation of CS surface amino groups under $\mathrm{pH}$ 4. This would lead to polymer swelling which enhances the network porosity and facilitates the drug release through diffusion ${ }^{47}$. At neutral $\mathrm{pH}$, the amino groups are uncharged and the CS molecular chains return to a more compact mode and the diffusion process is suppressed. ${ }^{48}$ Such $\mathrm{pH}$ responsive drug release profile is of particular interest for localized cancer treatment. On-demand release of anti-cancer drug can be triggered by the acidic tumor microenvironment, and the exposure to such drugs 
can be minimized under healthy conditions (neutral $\mathrm{pH}$ ) to reduce the associated side effects.

\subsubsection{Dual drug release from CS/GO hybrid coating}

While some researchers have demonstrated the feasibility of anti-cancer drug delivery from tissue scaffolds, ${ }^{49}$ scaffold systems offering controlled release of multiple therapeutic agents (e.g., pain relief, cancer drugs, antimicrobial agents, growth factors, etc) are scarce. The complex interplay between the materials, therapeutic payloads and the surrounding physiological environment present major challenges in the creation of a highly functional system. Nevertheless, such systems are highly desirable for applications such as the management of bone cancers, as multiple therapeutic agents, if supplied in an appropriate fashion, may offer higher treatment efficacy and promote tissue regeneration simultaneously. To investigate the feasibility of co-delivering multiple drugs from our scaffold, FL loaded GO and MB loaded CS were deposited onto the scaffold using the same LBL process resulting in (GO/FL-CS-MB) 20 samples. Results (Figure 4) show that dual release of FL and MB can be achieved by the hybrid coating, with the $\mathrm{pH}$ dependent release profile of FL being similar to the results shown in 2.2.1. MB release has been identified under both $\mathrm{pH}$ conditions, however, it is hard to distinguish the $\mathrm{pH}$ dependence of the $\mathrm{MB}$ release from the dual drug system from the UV-vis spectra. This may be because the MB characteristic peak has been shifted due to the interference of the strong signal from GO/FL across the whole wavelength range under neutral conditions (also see Figure 3(b)). 

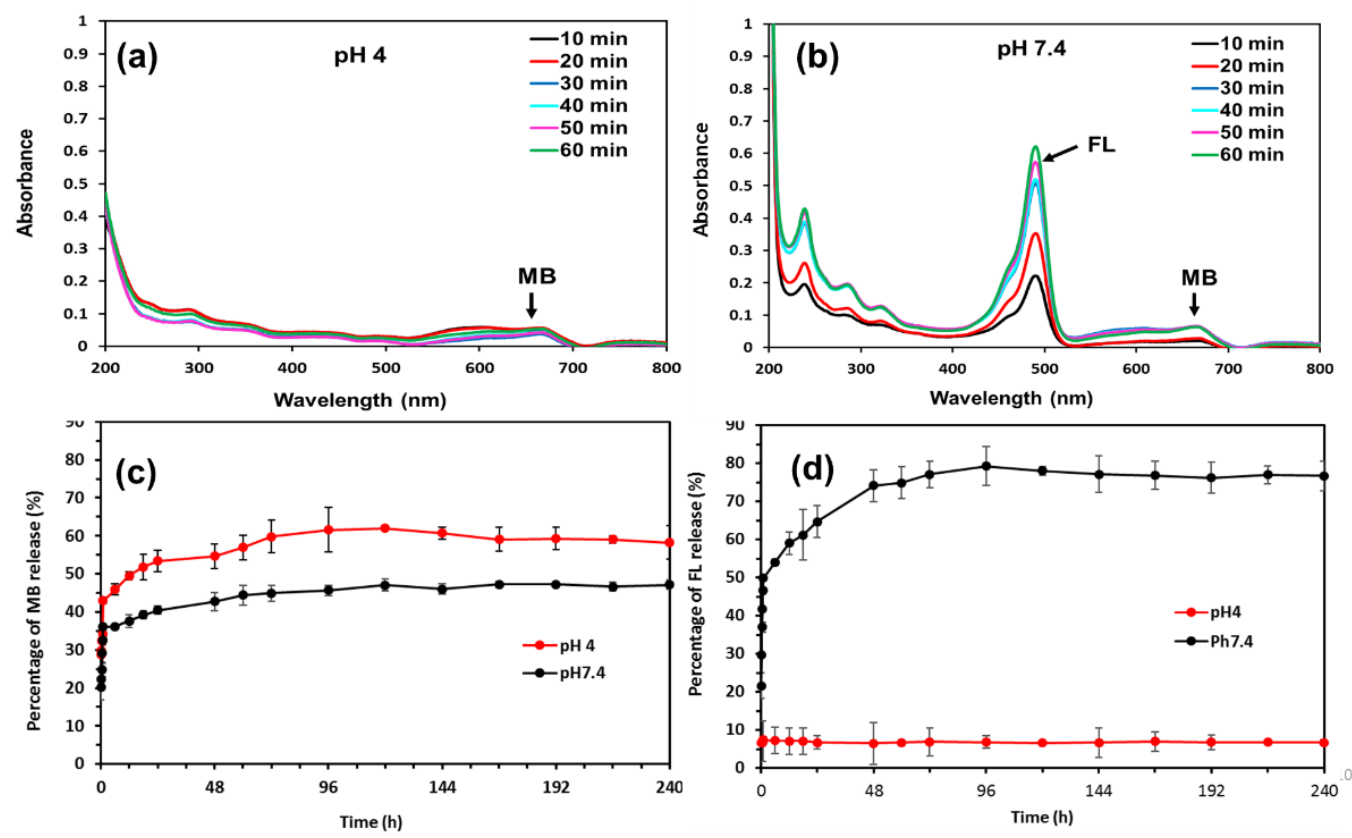

Figure 4. Drug release from (GO/FL-CS/MB) 20 scaffold in (a) $\mathrm{pH} 4$ and in (b) $\mathrm{pH} 7.4$ during the first hour, cumulative release (\%) of (c) MB and (d) FL for 240 hours.

\subsubsection{Anti-microbial properties}

Patients undergoing bone tumor surgeries are at high risk of postoperative deep infection caused by bacteria. Such complications present significant health challenges and usually require additional surgery, prolonged use of antibiotics, and delays of other scheduled treatments. ${ }^{51}$ To address this issue, we incorporated silver nanoparticles (AgNPs) into the CS/GO hybrid coating to serve as antimicrobial agents for a broad spectrum of Gramnegative and Gram-positive bacteria, including antibiotic-resistant strains. ${ }^{52,53}$ In addition, AgNPs have also demonstrated anti-cancer properties against various types of cancer cells such as human hepatoma cells ${ }^{54}$ lung cancer ${ }^{55}$ breast cancer $^{56}$ and cervical carcinoma, ${ }^{57}$ etc. The overall zeta potential of AgNPs loaded CS (CS/AgNPs) is $56.4 \mathrm{mv}$, see Figure 5 (a). The overall positive charge of the CS/AgNPs indicates it can still form a hybrid coating with the negatively charged GO through the previously described LBL process. Negatively charged AgNPs were loaded onto CS as described in the experimental section. The UV-vis spectra of CS/AgNPs overlaps with that of the AgNPs and the strong characteristic peaks at $398 \mathrm{~nm}$ 
indicates the successful loading of AgNPs (Figure 5 (b)). S.aureus (ATCC 6538) and E.coli (ATCC 11303) were used for antimicrobial evaluation. Figure 5 (c) shows that the AgNPs loaded scaffold displayed anti-bacterial ability against both S. aureus and E.coli. The number of bacteria drastically decreased $(68.85 \% \pm 2.87$ of S.aureus and $98.75 \pm 0.73 \%$ of E.coli) in the inoculum containing the AgNPs loaded scaffold (Figure 7(d)), confirming its effectiveness in antimicrobial applications.
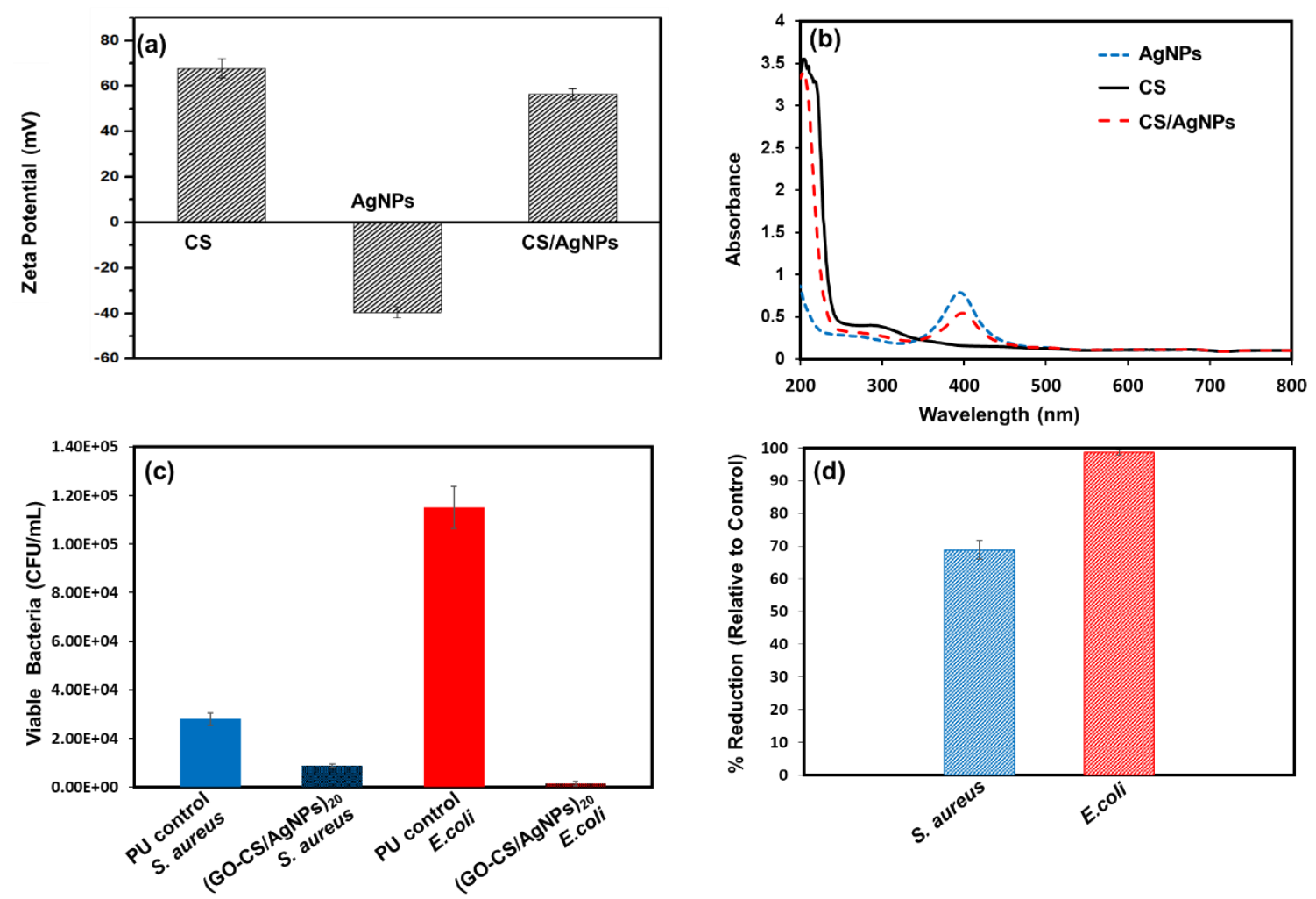

Figure 5. (a) zeta potential of as-prepared CS, AgNPs and CS/AgNPs; (b) UV-vis spectra of as-prepared CS, AgNPs and CS/AgNPs; (c) Viable S.aureus (CFU/mL) and E.coli remaining in the wells containing control PU and (GO-CS/AgNPs) 20 after 4 hours of incubation; (d) reduction of bacteria relative to the control PU.

\subsubsection{Photothermal property}

It is known that the GO exhibits strong light absorption ability in the near-infrared (700$900 \mathrm{~nm}$ ) region, ${ }^{58}$ and can effectively convert light energy into heat due to the delocalization of the electron states. ${ }^{58-60}$ Figure 6 shows the IR thermal images of PU control and (GO-CS $)_{20}$ 
with hybrid coatings under $\mathrm{CO}_{2}$ laser $(808 \mathrm{~nm}, 0.15 \mathrm{~mW})$ irradiation for up to $10 \mathrm{~min}$. The uncoated PU scaffold showed no temperature rise throughout the test period whereas the average temperature of the coated scaffold (at the point of irradiation) increased from $25^{\circ} \mathrm{C}$ to $45^{\circ} \mathrm{C}$ within 30s. The average temperature on (GO-CS $)_{20}$ scaffolds remained stable both in air and PBS solution throughout the rest of the test. It is possible to adjust the photothermal conversion efficiency of the GO containing scaffold through manipulating the GO concentration, the NIR power density and/or the size of the scaffold. ${ }^{38}$ The temperature of the scaffold under irradiation can be adjusted to $40-44{ }^{\circ} \mathrm{C}$, i.e., within the therapeutic window for cancer hyperthermia. ${ }^{61,62}$ With the NIR being able to penetrate tissues with sufficient intensity and high spatial precision, it is expected that our scaffold can be tailored to offer localized photothermal therapy when required. Additionally, photothermal effect has also be shown to facilitate anti-cancer drug release from graphene based nanocarriers, ${ }^{63}$ hence our hybrid coating modified scaffold could also serve as an ideal platform offering multi-modal therapy for bone cancer treatment with the option of stimuli induced drug release.

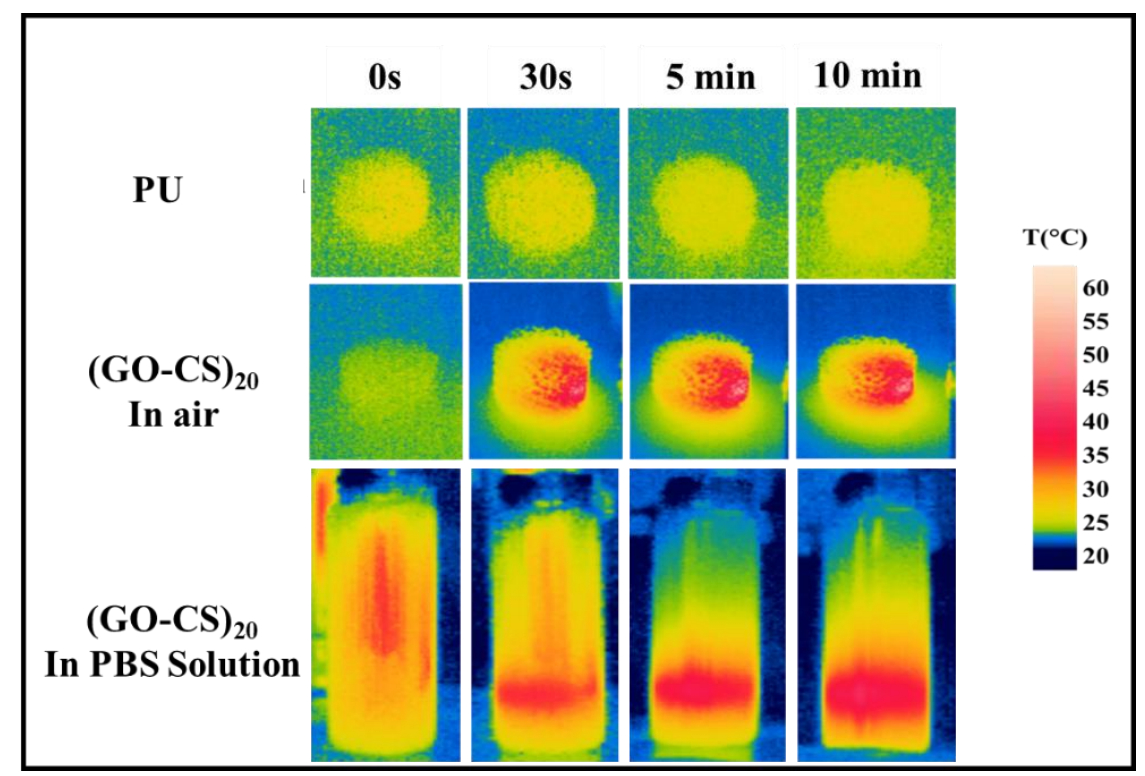

Figure 6. IR thermal images of (a) PU control and (GO-CS) 20 in (b) air and (c) PBS solution under $808 \mathrm{~nm}$ laser irradiation.

\subsubsection{In vitro cell behavior}

\section{Cell morphology}


SEM images in Figure 7A shows the degree of BMSCs adhesion on our scaffolds after $2 \mathrm{~h}$ incubation. The cells on the control group (PU scaffold), show more globular morphology with limited spreading despite abundant filopodia were found on the cell leading edge. In contrast, BMSCs on (GO-CS) 20 scaffold samples exhibited much better spreading featuring triangular, ellipsoidal or polygonal morphologies. Apparently, cells attached to the (GO-CS $)_{20}$ scaffold demonstrated more visible inter-connected lamellipodia, and the number of BMSCs on (GO-CS $)_{20}$ scaffolds are much higher than PU scaffolds, which suggests that (GO-CS $)_{20}$ favors better cell viability and stronger cell adherence.

The more clearly organized cytoskeletons were shown in the CLSM images in Figure 7B, which further confirms the BMSCs adhesion behavior on our scaffolds. Vinculin is a protein significantly involved in cell adhesion dynamics and, in particular, focal adhesion maturation, whereas cell adhesion is one of the most significant steps for cellular functions such as survival and differentiation. ${ }^{64}$ It is proposed integrin $\beta 1$ protein can mediate cell adhesion and integrate signals from a variety of cytokines and other growth factors. NELL-1(potent osteo-inductive factor) binding to integrin $\beta 1$ activates an intracellular signaling cascade that promotes cell adhesion, proliferation, and osteogenic differentiation. ${ }^{65}$ It can be seen that the expression of both integrin $\beta 1$ and vinculin manifested more abundant expression (much stronger fluorescent intensity) on the (GO-CS) 20 scaffold in comparison to the pure PU scaffold after $4 \mathrm{~h}$ of culturing.

The much stronger fluorescent intensity of integrin $\beta 1$ and vinculin expression on (GOCS) $)_{20}$ scaffold suggest the coated scaffold shows stronger cell adhesion than the PU control sample. ${ }^{65,66}$ 

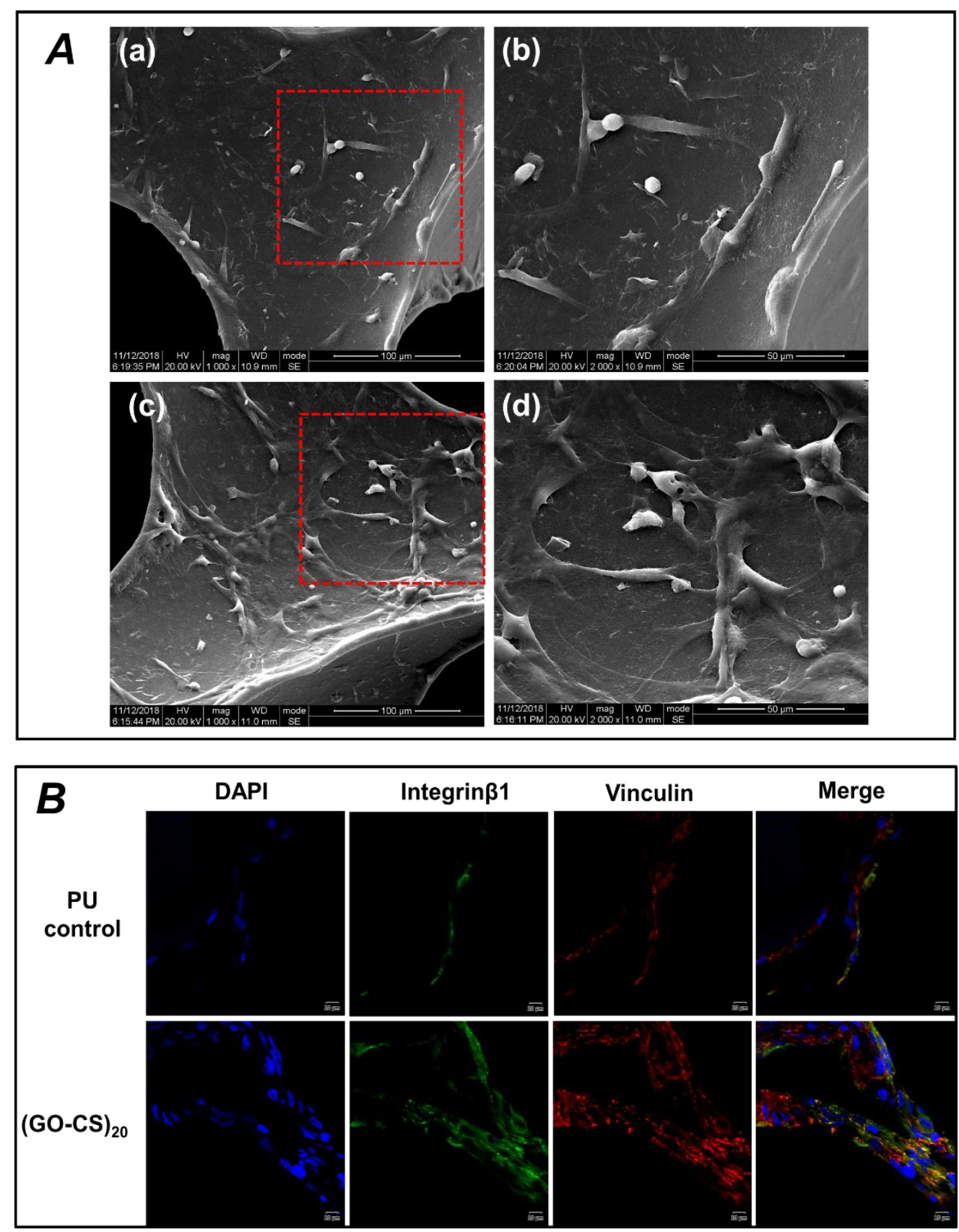

Figure 7. A: Initial cell adhesion after incubation for $2 \mathrm{~h}$, (a) PU scaffold, (c) (GO-CS $)_{20}$ scaffold, B: The expression of integrin $\beta 1$ and vinculin detected by immunofluorescence assay, and the merged images of integrin $\beta 1$ (green), vinculin (red) and DAPI nuclei on scaffold. $\operatorname{Bar}=10 \mu \mathrm{m}$.

The proliferation of cells was qualitatively determined by CCK-8 assay, see Figure 8. All 
groups exhibit gradually increased OD values from day 1 to day 5, suggesting a continuous cell proliferation in each group. It can be seen that both PU scaffold and (GO-CS) 20 scaffold can induce cell proliferation over a 5-day period, further confirming our multi-functional scaffolds exhibit excellent biocompatibility and can be used for potential in vivo applications.

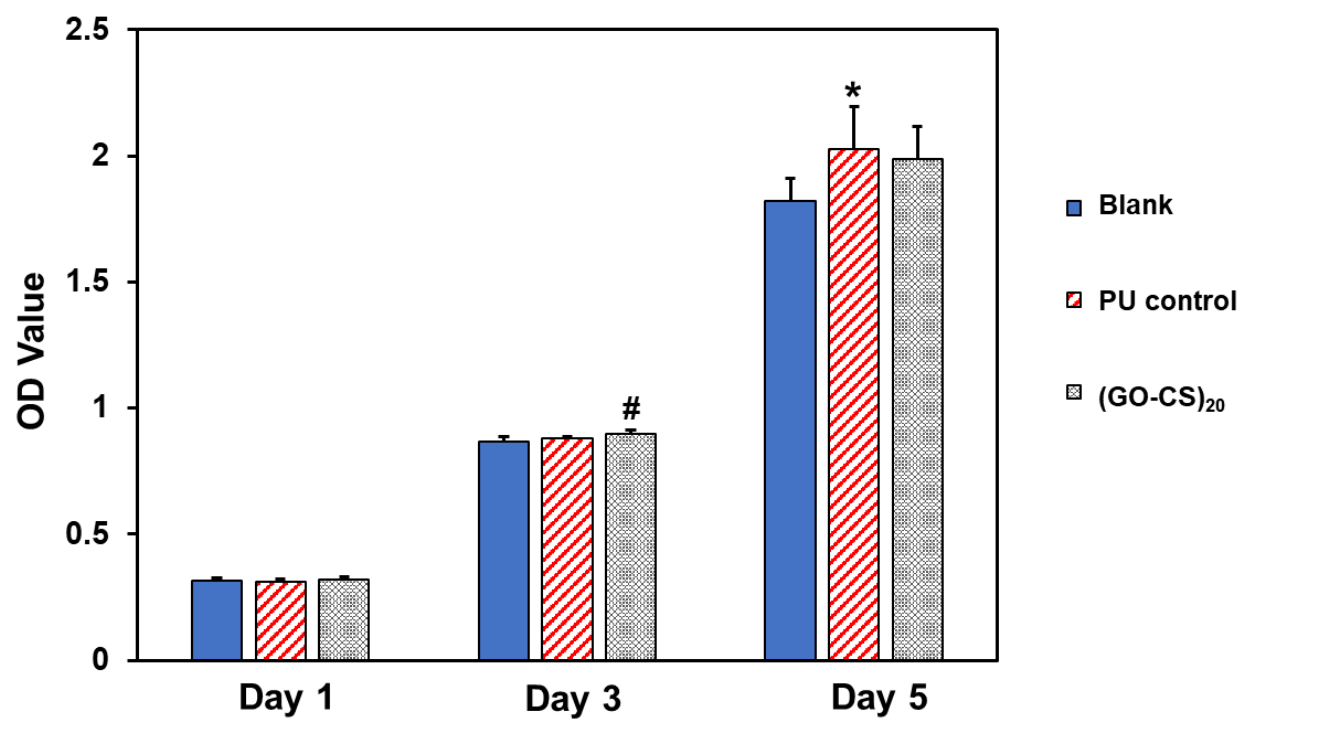

Figure 8. CCK-8 assay proliferation after 1, 3 and 5 days of culture, $\mathrm{n}=5$ per group; $(*), p<$ 0.05 when compared with blank group, $(\#), p<0.05$ when compared with PU control group.

\section{Conclusion}

In this study, we have created bio-compatible porous tissue scaffolds with a unique nanoscale hybrid coating consisting of alternating layers of graphene oxide nanosheets and chitosan, through a nature-inspired layer-by-layer assembly process. The coating is electrically conductive and can impart improved thermal stability and mechanical properties to the PU substrate. Results show that our coated scaffold can offer $\mathrm{pH}$-dependent delivery of multiple therapeutic agents, as well as anti-microbial properties and localized photothermal therapy. The structure / functionality of the scaffold can be tailored through nanoscale control over the coating architectures according to the specific needs of the diseases. We envisage our method can be generalized to create functional surfaces for a wide range of hard/soft scaffold materials 
(e.g., metals, ceramics, polymers, 3D printed structures, composites, etc) and the coating can be loaded with other therapeutic agents such as practical cancer drugs and growth factors. With modern healthcare technology paving its way towards customized solutions for best patient outcomes, our multi-functional scaffold can potentially revolutionize the way for future multimodal therapeutics in the biomedical field.

\section{Experimental Methods}

\subsection{Fabrication of the multi-layer CS/GO hybrid coating}

Open-cell polyurethane (PU) foam with 30 pores per inch (PPI) was supplied by the Foam Shop Ltd, UK. Graphene oxide (GO, 2mg/mL, dispersion in $\mathrm{H}_{2} \mathrm{O}$ ) and Chitosan (CS, low molecular weight 50,000-190,000 Da, 75-85\% deacetylated) were purchased from Sigma Aldrich. The PU foam was cut into cylindrical foam templates $(12.7 \mathrm{~mm}$ in diameter and 10 $\mathrm{mm}$ in height) for further coating deposition. As illustrated by Scheme 1, PU foam templates were first immersed in $1 \mathrm{M} \mathrm{NaOH}$ solution for $10 \mathrm{~min}$, thoroughly rinsed with DI water, and dried in oven at $37^{\circ} \mathrm{C}$ for 12 hours prior to coating deposition. The foam templates were then immersed in polyethylenimine (PEI, Sigma Aldrich branched, average Mw 25000, $0.01 \mathrm{M}$ ) solution for $60 \mathrm{~min}$ to generate a primer layer followed by rinsing with DI water. The LBL coating was formed by immersing the foam templates into GO $(2 \mathrm{mg} / \mathrm{ml})$ and $0.5 \%$ wt CS solution (in $2 \%(\mathrm{v} / \mathrm{v})$ acetic acid aqueous solution) alternatively. The immersion time was 30 min in each solution, and the samples were rinsed and dried between immersions. This procedure was repeated until a desired number of layers was achieved. The as-prepared specimens were denoted as $(\mathrm{GO}-\mathrm{CS})_{\mathrm{n}}$, where $\mathrm{n}$ indicates the number of GO/CS bilayers formed on the PU templates.

\subsection{Loading of model drugs}

Fluorescein sodium (FL, $1.2 \mathrm{wt} \%$, Sigma Aldrich) and methylene blue (MB, Sigma Aldrich) were used as model drugs for the preparation of drug release test. GO nanosheets were loaded with FL by adding $1.2 \mathrm{wt} \%$ FL to $2 \mathrm{mg} / \mathrm{mL}$ GO aqueous solution, stirring overnight at 
room temperature, then sonicating for $48 \mathrm{~h}$. Unbound FL was removed through centrifugation (3500 rpm for $6 \mathrm{~h}$ ). The FL loaded GO (GO/FL) was then re-dispersed in DI water by sonication for $12 \mathrm{~h}$. The final FL loading on GO is $32.4 \%$ according to TGA analysis. MB loaded CS (CS/MB) was obtained by adding MB into prepared CS solution $(0.2 \mathrm{mg} / \mathrm{mL})$ and stirred under $37^{\circ} \mathrm{C}$ for $8 \mathrm{~h}$. Following similar LBL assembly technique shown in Scheme. 1, three types of drug loaded samples were prepared, namely $(\mathrm{GO} / \mathrm{FL}-\mathrm{CS})_{\mathrm{n}},(\mathrm{GO}-\mathrm{CS} / \mathrm{MB})_{\mathrm{n}}$ and $(\mathrm{GO} / \mathrm{FL}-$ $\mathrm{CS} / \mathrm{MB})_{\mathrm{n}}$, where $\mathrm{n}$ denotes the number of GO/CS bi-layers. Silver nanoparticles (AgNPs, 10nm diameter, Sigma Aldrich) were added into the CS solution $(0.02 \mathrm{mg} / \mathrm{mL})$ and sonicated for $4 \mathrm{~h}$, and used to prepare (GO-CS/Ag) $)_{\mathrm{n}}$ samples.

\subsection{Characterization}

The surface charge of GO and CS were determined by zeta potential analysis using a Malvern Zetasizer Nano instrument with a $633 \mathrm{~nm}$ laser. All data were averaged over three measurements. Transmission FTIR was conducted under ambient conditions using a Perkin Elmer spectrometer. Resolution was set to $4 \mathrm{~cm}^{-1}$. Thirty-six scans per sample were recorded, averaged and corrected against the spectrum of attenuated total reflection crystals as background. Thermogravimetric analysis (TGA, TA Instruments SDT-Q600) was carried out over a temperature range 40 to $600{ }^{\circ} \mathrm{C}$ (heating rate $10{ }^{\circ} \mathrm{C} \mathrm{min}^{-1}$ ) under a nitrogen atmosphere. The weight of all samples was kept within 9-13 mg in an open alumina pan. Scanning electron microscopy (SEM) images were taken with a JEOL JSM-7001 F4 microscope, using acceleration voltage in the range of $2-15 \mathrm{kV}$. Prior to SEM, samples were cryo-fractured in liquid nitrogen and sputter coated with gold. To better confirm the LBL coating thickness and its electrical conductivity, GO/CS hybrid coating has also been deposited onto clean $\mathrm{Si}$ substrate following the same LBL technique as described earlier. The thickness of deposited coating was then measuring using atomic force microscopy (AFM, Cypher ES system, Santa Barbara, USA) under tapping mode with AC160TS cantilevers. The electrical conductivity of the coating was measured using an Agilent 34450A51/2 digital multimeter following methods reported elsewhere ${ }^{39,67}$.

The release of model drugs was characterized using UV-Vis spectroscopy (Cary Win UV) 
under $\mathrm{pH} 7.4$ and $\mathrm{pH} 4$, respectively. The relative characteristic peak intensities $(450 \mathrm{~nm}$ for $\mathrm{FL}, 665 \mathrm{~nm}$ for $\mathrm{MB}$ ) were compared at different time interval to determine the drug release profile as a function of time. The photothermal effects of specimens were tested under $808 \mathrm{~nm}$ $\mathrm{CO}_{2}$ laser irradiation $\left(0.15 \mathrm{~W} \mathrm{~cm} \mathrm{~cm}^{-2}\right)$ in an ambient environment. The temperature of the specimen was monitored by an FLIR i60 thermal imaging camera, with images frequency of 9 $\mathrm{Hz}$ and IR resolution of $180 \times 180$ pixels. The thermal images were further analyzed by FLIR R\&D software. Quasi-static mechanical testing in compression was conducted using a Lloyd's LRX Tensilce Tester with 50N load cell following ASTM D1621-101 with non-standard specimen sizes $(12.7 \mathrm{~mm}$ diameter, $10 \mathrm{~mm}$ height). Specimens were deformed at a displacement rate of $0.5 \mathrm{~mm} \mathrm{~s}^{-1}$, within the linear elastic range (approximately $0.6 \mathrm{~mm}$ displacement). The elastic modulus of each samples was calculated as the slope of the stressstrain plot in the linear portion of the curve.

\subsection{Antibacterial testing}

Escherichia coli (E. coli) ATCC 11303 and Staphylococcus aureus (S. aureus) ATCC 6538 were chosen as standard bacteria and were incubated in Mueller-Hinton Broth medium. PU control and (GO-CS/AgNPs)5 samples were sterilized and placed in 24-well cultures. The bacteria were seeded on these samples with a concentration of $1 \times 10^{6} \mathrm{CFU} / \mathrm{mL}$ plate in an incubator at $37^{\circ} \mathrm{C}$ for $4 \mathrm{~h}$. After 4 hours, $200 \mu \mathrm{L}$ of inoculum within the wells was removed by pipette, serially diluted and spread on Mueller-Hinton agar plates and incubated at $37{ }^{\circ} \mathrm{C}$ overnight for the colonies count.

\subsection{In vitro studies}

\subsubsection{Isolation and culture of bone marrow stem cells}

Cell isolation and culture were conducted following the standards established by the Animal Research Committee of the State Key Laboratory of Oral Diseases and West China School of Stomatology, Sichuan University (WCHSIRB-D-2018-003). Briefly, bone marrow stem cells (BMSCs) were harvested and extracted from the femurs of 4-week-old male Sprague-Dawley rats (Animal Research Center, Sichuan University, China) and cells at passage 2-4 were used for the cell experiment in this study. 


\subsubsection{Cell morphology}

After 2 hours of cell incubation $\left(5 \times 10^{4}\right)$, the scaffold samples were rinsed three times with copious amount of PBS to remove unattached cells. The remaining cells were fixed with $2.5 \%$ glutaraldehyde (Sigma-Aldrich). The cell morphology was analyzed using SEM. More specifically, the cells were dehydrated in a series of ethanol solutions $(20 \%, 40 \%, 60 \%, 80 \%$, $90 \%$, and $100 \%$ ) and then gold sputtered for SEM analysis.

\subsubsection{Cell immunofluorescence staining}

Cell immunofluorescence staining, the expressions of integrin $\beta 1$, vinculin, on the experimental samples were detected by CLSM. After incubation with the samples for $4 \mathrm{~h}$, the cells were fixed in $4 \%$ paraformaldehyde, and permeabilized with $0.2 \%$ Triton X-100. Subsequently, 4', 6-diamidino-2-phenylindole (DAPI, company, country) and specific antibodies targeting protein of interest were added sequentially and co-incubated for $4 \mathrm{~h}$ as previously described. All procedure was completed in dark and all samples were observed using CLSM after thorough rinsing using PBS.

\subsubsection{Cell proliferation}

L929 cells (CCL-1) derived from normal subcutaneous areolar and adipose tissue of $\mathrm{C} 3 \mathrm{H} / \mathrm{An}$ mouse were obtained from American Type Culture Collection (ATCC) and the cells were used for general biocompatibility tests according to ISO10993. Cells were first cultured in low glucose DMEM (Dulbecco's modified Eagle's medium, Gibco Laboratories, Gaithersburg, USA) supplemented with 5\% fotal bovine serum (FBS) (Hyclone, Logan, UT, USA) and 1\% penicillin/streptomycin (Gibco Laboratories, Gaithersburg, MD, USA). Then the cells were incubated at $37{ }^{\circ} \mathrm{C}$ in a $5 \% \mathrm{CO}_{2}$ humidified atmosphere. The proliferation rate of L929 cells was estimated using a cell counting kit (CCK-8; DOJINDO Laboratories, Japan) assay. 


\subsection{Statistical analyses}

Data were expressed as mean \pm standard deviation (SD) and analyzed by using the SPSS 16.0 software (SPSS Inc., USA). Significant differences among the groups were assessed using the one-way analysis of variance (ANOVA) followed by the least significant difference (LSD) multiple comparison test. Statistical significance was defined as $p<0.05$.

\section{Associated content}

Supporting Information

The Supporting Information is available free of charge on the XXX website at XXX.

\section{Author information}

Corresponding Authors: Dan Sun, Songsong Zhu

Tel: +44 (0) 2890974701

E-mail: d.sun@qub.ac.uk; zss_1977@163.com

\section{Author Contributions}

$\dagger$ N. Jiang and Z. Guo contributed equally to this work.

Z. Guo and D. Sun conceived ideas of this work. Z. Guo carried out all experimental work in relation to materials synthesis and characterization. N. Jiang performed all biology related work. Jessica Moore carried out all bacterial related work. C. Rafferty and G. Sarri contributed to the laser related tests. All authors contributed to the analysis of data and result discussion as well as the writing of the manuscript.

\section{Acknowledgements}

The authors of this work would like to thank the funding support from the Engineering and Physical Science Research Council (EPSRC, EP/P00394X/1, EP/P010059/1), National Natural Science Foundation of China (No. 51673131), National Key Research and Development Program of China (2016YFA0201703/2016YFA0201700), and China 
Scholarship Council (201606240059).

Competing Interests: The authors declare that they have no competing interests.

\section{Reference}

(1) Miller, K. D.; Siegel, R. L.; Lin, C. C.; Mariotto, A. B.; Kramer, J. L.; Rowland, J. H.; Stein, K. D.; Alteri, R.; Jemal, A. Cancer Treatment and Survivorship Statistics, 2016. CA. Cancer J. Clin. 2016, 66 (4), 271-289.

(2) Wong, B. S.; Teoh, S.-H.; Kang, L. Polycaprolactone Scaffold as Targeted Drug Delivery System and Cell Attachment Scaffold for Postsurgical Care of Limb Salvage. Drug Deliv. Transl. Res. 2012, 2 (4), 272-283.

(3) Palamà, I. E.; Arcadio, V.; D’Amone, S.; Biasiucci, M.; Gigli, G.; Cortese, B. Therapeutic PCL Scaffold for Reparation of Resected Osteosarcoma Defect. Sci. Rep. 2017, 7 (1), 12672.

(4) Zhang, J.; Zhao, S.; Zhu, M.; Zhu, Y.; Zhang, Y.; Liu, Z.; Zhang, C. 3D-Printed Magnetic $\mathrm{Fe}_{3} \mathrm{O}_{4} / \mathrm{MBG} / \mathrm{PCL}$ Composite Scaffolds with Multifunctionality of Bone Regeneration, Local Anticancer Drug Delivery and Hyperthermia. J. Mater. Chem. B 2014, 2 (43), 7583-7595.

(5) Zhang, J.; Li, J.; Kawazoe, N.; Chen, G. Composite Scaffolds of Gelatin and Gold Nanoparticles with Tunable Size and Shape for Photothermal Cancer Therapy. J. Mater. Chem. B 2017, 5 (2), 245-253.

(6) Wang, X.; Li, T.; Ma, H.; Zhai, D.; Jiang, C.; Chang, J.; Wang, J.; Wu, C. A 3DPrinted Scaffold with MoS2 Nanosheets for Tumor Therapy and Tissue Regeneration. NPG Asia Mater. 2017, 9 (4), e376-e376.

(7) Dang, W.; Li, T.; Li, B.; Ma, H.; Zhai, D.; Wang, X.; Chang, J.; Xiao, Y.; Wang, J.; Wu, C. A Bifunctional Scaffold with CuFeSe2 Nanocrystals for Tumor Therapy and Bone Reconstruction. Biomaterials 2018, 160, 92-106.

(8) Ma, H.; Jiang, C.; Zhai, D.; Luo, Y.; Chen, Y.; Lv, F.; Yi, Z.; Deng, Y.; Wang, J.; Chang, J.; et al. A Bifunctional Biomaterial with Photothermal Effect for Tumor Therapy and Bone Regeneration. Adv. Funct. Mater. 2016, 26 (8), 1197-1208. 
(9) Kucińska-Lipka, J.; Gubanska, I.; Skwarska, A. Microporous Polyurethane Thin Layer as a Promising Scaffold for Tissue Engineering. Polymers (Basel). 2017, 9 (12), 277.

(10) Fernando, S.; McEnery, M.; Guelcher, S. A. Polyurethanes for Bone Tissue Engineering. In Advances in Polyurethane Biomaterials; Elsevier, 2016; pp 481-501.

(11) Wolska, A.; Godzikiewicz, M.; Ryszkowska, J. Thermal and Mechanical Behaviour of Flexible Polyurethane Foams Modified with Graphite and Phosphorous Fillers. 2012.

(12) Kucińska-Lipka, J.; Gubańska, I.; Janik, H. Gelatin-Modified Polyurethanes for Soft Tissue Scaffold. ScientificWorldJournal. 2013, 2013, 450132.

(13) Li, M.; Yang, X.; Ren, J.; Qu, K.; Qu, X. Using Graphene Oxide High NearInfrared Absorbance for Photothermal Treatment of Alzheimer's Disease. Adv. Mater. 2012, 24 (13), 1722-1728.

(14) Luo, X.; Weaver, C. L.; Tan, S.; Cui, X. T. Pure Graphene Oxide Doped Conducting Polymer Nanocomposite for Bio-Interfacing. J. Mater. Chem. B 2013, 1 (9), 1340.

(15) Yang, K.; Feng, L.; Shi, X.; Liu, Z. Nano-Graphene in Biomedicine: Theranostic Applications. Chem. Soc. Rev. 2013, 42 (2), 530-547.

(16) Hung, A. H.; Holbrook, R. J.; Rotz, M. W.; Glasscock, C. J.; Mansukhani, N. D.; Macrenaris, K. W.; Manus, L. M.; Duch, M. C.; Dam, K. T.; Hersam, M. C.; et al. Graphene Oxide Enhances Cellular Delivery of Hydrophilic Small Molecules by Co-Incubation. ACS Nano 2014, 8 (10), 10168-10177.

(17) Weaver, C. L.; Larosa, J. M.; Luo, X.; Cui, X. T. Electrically Controlled Drug Delivery from Graphene Oxide Nanocomposite Films. ACS Nano 2014, 8 (2), 1834-1843.

(18) Akhavan, O.; Ghaderi, E.; Shirazian, S. A.; Rahighi, R. Rolled Graphene Oxide Foams as Three-Dimensional Scaffolds for Growth of Neural Fibers Using Electrical Stimulation of Stem Cells. Carbon N. Y. 2016, 97, 71-77. 
(19) Holt, B. D.; Wright, Z. M.; Arnold, A. M.; Sydlik, S. A. Graphene Oxide as a Scaffold for Bone Regeneration. Wiley Interdiscip. Rev. Nanomedicine Nanobiotechnology 2017, 9 (3), 1-18.

(20) Rao, W.; Wang, H.; Han, J.; Zhao, S.; Dumbleton, J.; Agarwal, P.; Zhang, W.; Zhao, G.; Yu, J.; Zynger, D. L.; et al. Chitosan-Decorated DoxorubicinEncapsulated Nanoparticle Targets and Eliminates Tumor Reinitiating Cancer Stem-like Cells. ACS Nano 2015, 9 (6), 5725-5740.

(21) Rajam, M.; Pulavendran, S.; Rose, C.; Mandal, A. B. Chitosan Nanoparticles as a Dual Growth Factor Delivery System for Tissue Engineering Applications. Int. J. Pharm. 2011, 410 (1-2), 145-152.

(22) Sobhani, Z.; Mohammadi Samani, S.; Montaseri, H.; Khezri, E. Nanoparticles of Chitosan Loaded Ciprofloxacin: Fabrication and Antimicrobial Activity. Adv. Pharm. Bull. 2017, 7 (3), 427-432.

(23) Finnemore, A.; Cunha, P.; Shean, T.; Vignolini, S.; Guldin, S.; Oyen, M.; Steiner, U. Biomimetic Layer-by-Layer Assembly of Artificial Nacre. Nat. Commun. 2012, 3 (1), 966.

(24) Kaur, G.; Adhikari, R.; Cass, P.; Bown, M.; Gunatillake, P. Electrically Conductive Polymers and Composites for Biomedical Applications. RSC Adv. 2015, 5 (47), 37553-37567.

(25) Martins, A. M.; Eng, G.; Caridade, S. G.; Mano, J. F.; Reis, R. L.; VunjakNovakovic, G. Electrically Conductive Chitosan/Carbon Scaffolds for Cardiac Tissue Engineering. Biomacromolecules 2014, 15 (2), 635-643.

(26) Roth, B. J. Electrical Conductivity Values Used with the Bidomain Model of Cardiac Tissue. IEEE Trans. Biomed. Eng. 1997, 44 (4), 326-328.

(27) Weaver, C. L.; LaRosa, J. M.; Luo, X.; Cui, X. T. Electrically Controlled Drug Delivery from Graphene Oxide Nanocomposite Films. ACS Nano 2014, 8 (2), 1834-1843.

(28) Martins, A. M.; Eng, G.; Caridade, S. G.; Mano, J. F.; Reis, R. L.; VunjakNovakovic, G. Electrically Conductive Chitosan/Carbon Scaffolds for Cardiac 
Tissue Engineering. Biomacromolecules 2014, 15 (2), 635-643.

(29) Akhtari, M.; Emin, D.; Ellingson, B. M.; Woodworth, D.; Frew, A.; Mathern, G.

W. Measuring the Local Electrical Conductivity of Human Brain Tissue. J. Appl. Phys. 2016, 119 (10).

(30) Lu, Y.; Lyu, H.; Richardson, A. G.; Lucas, T. H.; Kuzum, D. Flexible Neural Electrode Array Based-on Porous Graphene for Cortical Microstimulation and Sensing. Sci. Rep. 2016, 6 (1), 33526.

(31) Xiong, W.; Zhou, Y. S.; Hou, W. J.; Jiang, L. J.; Gao, Y.; Fan, L. S.; Jiang, L.; Silvain, J. F.; Lu, Y. F. Direct Writing of Graphene Patterns on Insulating Substrates under Ambient Conditions. Sci. Rep. 2014, 4, 1-6.

(32) McFee, R.; Rush, S. Qualitative Effects of Thoracic Resistivity Variations on the Interpretation of Electrocardiograms: The Low Resistance Surface Layer. Am. Heart J. 1968, 76 (1), 48-61.

(33) Potse, M.; Dubé, B.; Vinet, A. Cardiac Anisotropy in Boundary-Element Models for the Electrocardiogram. Med. Biol. Eng. Comput. 2009, 47 (7), 719-729.

(34) Zhu, X.; Feng, W.; Chang, J.; Tan, Y.-W.; Li, J.; Chen, M.; Sun, Y.; Li, F. Temperature-Feedback Upconversion Nanocomposite for Accurate Photothermal Therapy at Facile Temperature. Nat. Commun. 2016, 7, 10437.

(35) Sharma, D.; Kanchi, S.; Sabela, M. I.; Bisetty, K. Insight into the Biosensing of Graphene Oxide: Present and Future Prospects. Arab. J. Chem. 2016, 9 (2), 238-261.

(36) Kim, J.; Kim, H. D.; Park, J.; Lee, E.-S.; Kim, E.; Lee, S. S.; Yang, J.-K.; Lee, Y.-S.; Hwang, N. S. Enhanced Osteogenic Commitment of Murine Mesenchymal Stem Cells on Graphene Oxide Substrate. Biomater. Res. 2018, $22,1$.

(37) Ghasemi-Mobarakeh, L.; Prabhakaran, M. P.; Morshed, M.; Nasr-Esfahani, M. H.; Baharvand, H.; Kiani, S.; Al-Deyab, S. S.; Ramakrishna, S. Application of Conductive Polymers, Scaffolds and Electrical Stimulation for Nerve Tissue Engineering. J. Tissue Eng. Regen. Med. 2011, 5 (4), e17-e35. 
(38) Justin, R.; Chen, B. Strong and Conductive Chitosan-reduced Graphene Oxide Nanocomposites for Transdermal Drug Delivery. J. Mater. Chem. B 2014, 2 (24), 3759.

(39) Gonçalves, G.; Vila, M.; Portolés, M.-T.; Vallet-Regi, M.; Gracio, J.; Marques, P. A. A. P. Nano-Graphene Oxide: A Potential Multifunctional Platform for Cancer Therapy. Adv. Healthc. Mater. 2013, 2 (8), 1072-1090.

(40) Xu, Y.; Sheng, K.; Li, C.; Shi, G. Self-Assembled Graphene Hydrogel via a One-Step Hydrothermal Process.

(41) Kim, Y.-H.; Tabata, Y. Full-TextDual-Controlled Release System of Drugs for Bone Regeneration. Adv. Drug Deliv. Rev. 2015, 94, 28-40.

(42) Shih, C.-J.; Lin, S.; Sharma, R.; Strano, M. S.; Blankschtein, D. Understanding the PH-Dependent Behavior of Graphene Oxide Aqueous Solutions: A Comparative Experimental and Molecular Dynamics Simulation Study. Langmuir 2012, 28 (1), 235-241.

(43) Li, D.; Müller, M. B.; Gilje, S.; Kaner, R. B.; Wallace, G. G. Processable Aqueous Dispersions of Graphene Nanosheets. Nat. Nanotechnol. 2008, 3 (2), 101-105.

(44) Casalini, T.; Salvalaglio, M.; Perale, G.; Masi, M.; Cavallotti, C. Diffusion and Aggregation of Sodium Fluorescein in Aqueous Solutions. J. Phys. Chem. $B$ 2011, 115 (44), 12896-12904.

(45) Kim, Y.-H.; Tabata, Y. Dual-Controlled Release System of Drugs for Bone Regeneration. Adv. Drug Deliv. Rev. 2015, 94, 28-40.

(46) Mohaiyiddin, M. S.; Ong, H. L.; Othman, M. B. H.; Julkapli, N. M.; Villagracia, A. R. C.; Md. Akil, H. Swelling Behavior and Chemical Stability of Chitosan/Nanocellulose Biocomposites. Polym. Compos. 2018, 39, E561E572.

(47) Zheng, J. Energy Metabolism of Cancer: Glycolysis versus Oxidative Phosphorylation (Review). Oncology Letters. 2012.

(48) Milošev, I. Surface Treatments of Titanium with Antibacterial Agents for 
Implant Applications; 2016; pp 1-87.

(49) Palamm, I. E.; Arcadio, V.; D 'amone, S.; Biasiucci, M.; Gigli, G.; Cortese, B. Therapeutic PCL Scaffold for Reparation of Resected Osteosarcoma Defect.

(50) Han, U.; Seo, Y.; Hong, J. Effect of PH on the Structure and Drug Release Profiles of Layer-by-Layer Assembled Films Containing Polyelectrolyte, Micelles, and Graphene Oxide. Sci. Rep. 2016, 6 (1), 24158.

(51) Miwa, S.; Shirai, T.; Yamamoto, N.; Hayashi, K.; Takeuchi, A.; Tada, K.; Kajino, Y.; Inatani, H.; Higuchi, T.; Abe, K.; et al. Risk Factors for Postoperative Deep Infection in Bone Tumors. PLoS One 2017, 12 (11), e0187438.

(52) Rai, M.; Kon, K. V. Nanotechnology in Diagnosis, Treatment and Prophylaxis of Infectious Diseases; Rai, M., Kon, K., Eds.; 2015.

(53) Gao, Y.; Hassanbhai, A. M.; Lim, J.; Wang, L.; Xu, C. Fabrication of a Silver Octahedral Nanoparticle-Containing Polycaprolactone Nanocomposite for Antibacterial Bone Scaffolds. RSC Adv. 2017, 7 (17), 10051-10056.

(54) Kawata, K.; Osawa, M.; Okabe, S. In Vitro Toxicity of Silver Nanoparticles at Noncytotoxic Doses to HepG2 Human Hepatoma Cells. Environ. Sci. Technol. 2009, 43 (15), 6046-6051.

(55) Foldbjerg, R.; Dang, D. A.; Autrup, H. Cytotoxicity and Genotoxicity of Silver Nanoparticles in the Human Lung Cancer Cell Line, A549. Arch. Toxicol. 2011, $85(7), 743-750$.

(56) Gurunathan, S.; Han, J. W.; Eppakayala, V.; Jeyaraj, M.; Kim, J.-H. Cytotoxicity of Biologically Synthesized Silver Nanoparticles in MDA-MB-231 Human Breast Cancer Cells. Biomed Res. Int. 2013, 2013, 1-10.

(57) Vasanth, K.; Ilango, K.; MohanKumar, R.; Agrawal, A.; Dubey, G. P. Anticancer Activity of Moringa Oleifera Mediated Silver Nanoparticles on Human Cervical Carcinoma Cells by Apoptosis Induction. Colloids Surfaces $B$ Biointerfaces 2014, 117, 354-359.

(58) Li, M.; Yang, X.; Ren, J.; Qu, K.; Qu, X. Using Graphene Oxide High Near- 
Infrared Absorbance for Photothermal Treatment of Alzheimer's Disease. Adv. Mater. 2012, 24 (13), 1722-1728.

(59) Hashemi, M.; Omidi, M.; Muralidharan, B.; Tayebi, L.; Herpin, M. J.; Mohagheghi, M. A.; Mohammadi, J.; Smyth, H. D. C.; Milner, T. E. Layer-byLayer Assembly of Graphene Oxide on Thermosensitive Liposomes for PhotoChemotherapy. Acta Biomater. 2018, 65, 376-392.

(60) Robinson, J. T.; Tabakman, S. M.; Liang, Y.; Wang, H.; Sanchez Casalongue, H.; Vinh, D.; Dai, H. Ultrasmall Reduced Graphene Oxide with High NearInfrared Absorbance for Photothermal Therapy. J. Am. Chem. Soc 2011, 133, $6825-6831$.

(61) Jaque, D.; Maestro, L. M.; Rosal, B. del; Haro-Gonzalez, P.; Benayas, A.; Plaza, J. L.; Rodríguez, E. M.; Solé, J. G. Nanoparticles for Photothermal Therapies. Nanoscale 2014, 6 (16), 9494-9530.

(62) Schildkopf, P.; Ott, O. J.; Frey, B.; Wadepohl, M.; Sauer, R.; Fietkau, R.; Gaipl, U. S. Biological Rationales and Clinical Applications of Temperature Controlled Hyperthermia--Implications for Multimodal Cancer Treatments. Curr. Med.

Chem. 2010, 17 (27), 3045-3057.

(63) Zeng, Y.; Yang, Z.; Li, H.; Hao, Y.; Liu, C.; Zhu, L.; Liu, J.; Lu, B.; Li, R. Multifunctional Nanographene Oxide for Targeted Gene-Mediated Thermochemotherapy of Drug-Resistant Tumour. Sci. Rep. 2017, 7, 43506.

(64) Jiang, N.; Guo, Z.; Sun, D.; Li, Y.; Yang, Y.; Chen, C.; Zhang, L.; Zhu, S. Promoting Osseointegration of Ti Implants through Micro/Nanoscaled Hierarchical Ti Phosphate/Ti Oxide Hybrid Coating. ACS Nano 2018, 12 (8), 7883-7891.

(65) Pakvasa, M.; Alverdy, A.; Mostafa, S.; Wang, E.; Fu, L.; Li, A.; Oliveira, L.; Athiviraham, A.; Lee, M. J.; Wolf, J. M.; et al. Neural EGF-like Protein 1 (NELL1): Signaling Crosstalk in Mesenchymal Stem Cells and Applications in Regenerative Medicine. Genes Dis. 2017, 4 (3), 127-137.

(66) Seo, C. H.; Jeong, H.; Furukawa, K. S.; Suzuki, Y.; Ushida, T. The Switching 
of Focal Adhesion Maturation Sites and Actin Filament Activation for MSCs by Topography of Well-Defined Micropatterned Surfaces. Biomaterials 2013, 34 (7), 1764-1771.

(67) Orecchioni, M.; Cabizza, R.; Bianco, A.; Delogu, L. G. Graphene as Cancer Theranostic Tool: Progress and Future Challenges. Theranostics 2015, 5 (57), 710-723. 